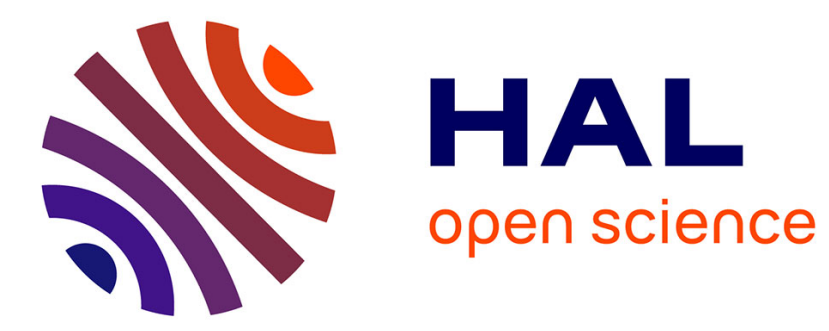

\title{
Nonlinear Polarization Dynamics of VCSEL with Frequency Comb Injection
}

Yaya Doumbia, Delphine Wolfersberger, Krassimir Panajotov, Marc

Sciamanna

\section{- To cite this version:}

Yaya Doumbia, Delphine Wolfersberger, Krassimir Panajotov, Marc Sciamanna. Nonlinear Polarization Dynamics of VCSEL with Frequency Comb Injection. Advanced Photonics Congress, Jul 2021, Montreal (virtual), Canada. hal-03229285

\section{HAL Id: hal-03229285 \\ https://hal.science/hal-03229285}

Submitted on 18 May 2021

HAL is a multi-disciplinary open access archive for the deposit and dissemination of scientific research documents, whether they are published or not. The documents may come from teaching and research institutions in France or abroad, or from public or private research centers.
L'archive ouverte pluridisciplinaire $\mathbf{H A L}$, est destinée au dépôt et à la diffusion de documents scientifiques de niveau recherche, publiés ou non, émanant des établissements d'enseignement et de recherche français ou étrangers, des laboratoires publics ou privés. 


\title{
Nonlinear Polarization Dynamics of VCSEL with Frequency Comb Injection
}

\author{
Yaya Doumbia ${ }^{1,2, *}$, Delphine Wolfersberger ${ }^{1,2}$, Krassimir Panajotov, ${ }^{3,4}$ and Marc \\ Sciamanna 1,2 \\ ${ }^{1}$ Chaire Photonique, CentraleSupélec, LMOPS, 2 Rue Edouard Belin 57070 Metz, France \\ ${ }^{2}$ Université de Lorraine, CentraleSupélec, LMOPS, 2 Rue Edouard Belin 57070 Metz, France \\ ${ }^{3}$ Brussels Photonics Group (B-PHOT), Vrije Universiteit Brussel, Brussels, Belgium \\ ${ }^{4}$ Institute of Solid State Physics, Bulgarian Academy of Sciences, Sofia, Bulgaria \\ *yaya.doumbia@centralesupelec.fr
}

\begin{abstract}
:
We propose a new approach of broad frequency comb generation by exploiting the polarization dynamics in a VCSEL induced by parallel frequency comb injection. Polarization mode competition leads to two tunable combs with orthogonal polarization. (C) 2021 The Author(s)
\end{abstract}

\section{Introduction}

Vertical-Cavity Surface-Emitting Lasers (VCSELs) have attracted significant attention due to their advantage of compactness, low cost, and low energy consumption and opens the possibility of mass production. VCSELs can show interesting polarization dynamics under optical injection [1]. It has been recently demonstrated that VCSEL can show complex polarization dynamics even in free running [2]. Recent experimental and theoretical analysis of polarization dynamics of VCSEL demonstrated the possibility of generation of two polarization comb under gain switching [3]. The quality of these combs obtained by gain switching depends strongly on the modulation frequency. The polarization combs properties have been found to improve with optical injection into the comb. The opposite situation in which a single-mode VCSEL is injected with an OFC has not been addressed so far. Recently, Optical frequency comb (OFC) injection in a diode laser has attracted significant attention [4,5].

In this work, we analyze the polarization dynamics of a VCSEL subject to a narrow OFC injection. More specifically, we exploit the polarization dynamics of a VCSEL to generate a broad OFC with one or two polarization.

\section{Experimental setup}
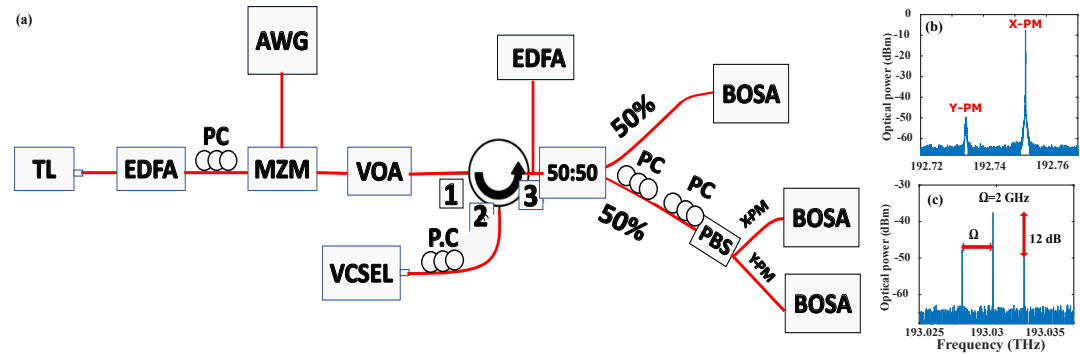

Fig. 1. (a) set up for OFC injection into a VCSEL. TL: Tunable Laser, EDFA: amplifier, PC: Polarization Controller, AWG: Arbitrary Waveform Generator, MZM: Mach-Zehnder Modulator, VOA: Variable Optical Attenuator, BOSA: Brillouin Optical Spectrum Analyser. Figure 1 (b) and (c) shows the optical spectra of the free-running VCSEL and the injected comb, respectively.

Figure 1 (a) shows the setup for an OFC injection. The tunable laser output is amplified with an amplifier (EDFA). Electric signal modulation is generated by an Arbitrary Waveform Generator (AWG) and sent to the RF port of a Mach-Zehnder Modulator (MZM). An optical comb with 3 optical frequency lines is generated in the output of the MZM ( see Fig. 1 (c)). The comb's optical power is controlled using a variable optical attenuator (VOA). The injected laser is a single-mode VCSEL with the dominant (X-PM) and depressed (Y-PM) polarization mode, respectively ( see Fig. 1 (b)). The optical injection is performed through a fiber circulator. The injected laser dynamics are analyzed with a BOSA. A Polarized beam splitter (PBS) is used to separately observe the dynamics 
of each polarization mode. The polarization controllers (PC) allows to align the laser polarization with the MZM, the VCSEL polarization with the axis of a (PBS), and to control the polarization of the injected light.

\section{Results}

Figure 2 (a) and (b) shows the optical spectra when the polarization of the injected OFC is turned to be parallel to that of the VCSEL. When varying the detuning for fixed injected power $P_{i n j}$, the VCSEL output shows a timeperiodic dynamics corresponding to an OFC with the same comb spacing as the injected comb in X-polarization mode (X-PM) (Fig. 2 (a)). When we keep varying the detuning, the VCSEL bifurcates to more complex dynamics in both polarization modes accompanied by an overall OFC generation as shown in Fig. 2 (b). We checked that
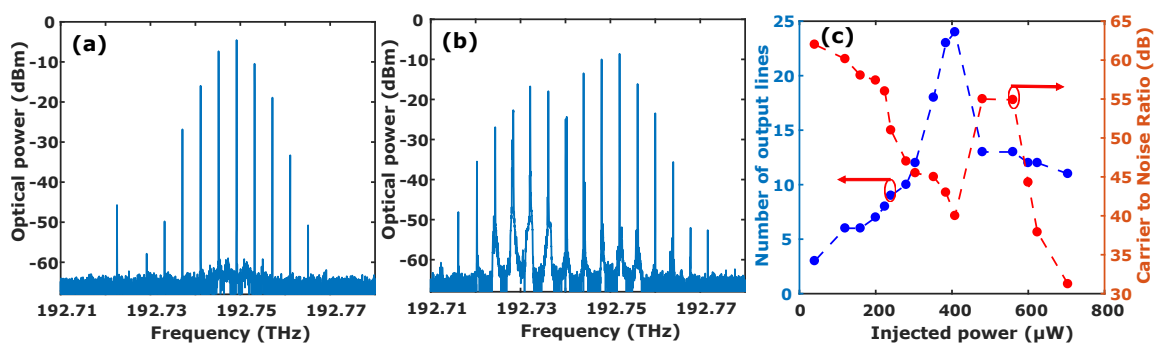

Fig. 2. (a), (b) optical spectra of VCSEL output for fixed comb spacing to $\Omega=4 \mathrm{GHz}$. (a) and (b) are obtained for $P_{i n j}=432.8 \mu \mathrm{W}$ and detuning $\Delta v=-2.6 \mathrm{GHz}$ and $\Delta v=-5.4 \mathrm{GHz}$, respectively.

(c) OFC performance analysis for fixed $\Delta v=-0.9 \mathrm{GHz}$.

this OFC is formed by two micro-OFC with orthogonal polarization modes.

Figure 2 (c) analyzes the performance of the combs when varying the injected power for fixed detuning $\Delta v=$ $-0.9 \mathrm{GHz}$. We qualify the comb quality by the number of lines (bandwidth) and Carrier to Noise Ratio (CNR). The CNR is the difference between the maximum amplitude in the optical spectrum and the noise level in $\mathrm{dB}$. When increasing $P_{i n j}$, the number of lines increases to achieve a maximum of around $P_{i n j}=400 \mu \mathrm{W}$ while the CNR decreases. When varying the injection parameters, our data shows the possibility to control the comb spacing through harmonic OFC generation. Harmonic OFCs with repetition rates of hundreds of $\mathrm{MHz}$ can be indeed demonstrated. Figure 2 (c) shows also that the best CNR is found for low $P_{i n j}$. We also check that the performance of the OFC dynamics improves with the bias current.

Numerical simulations are performed to analyze the impact of birefringence of the VCSEL on the two polarization OFC dynamics. Interestingly, we found that the resulting number of lines increases with the birefringence. Our theoretical results also predict that the comb lines of each polarization mode are phase-locked.

\section{Conclusion}

We have shown that injection of an OFC into a VCSEL induces interesting polarization dynamics leading to very wideband OFCs. Optimization of the injection parameters allows achieving a resulting comb bandwidth up to 13 times that of the injected comb and a Carrier to Noise Ratio (CNR) up to $60 \mathrm{~dB}$. These results can be used for polarization division multiplexing for optical data communications and dual-comb spectroscopy.

Funding Chaire Photonique: Ministère de l'Enseignement supérieur, de la Recherche et de l'Innovation; Région Grand Est; Département Moselle; European Regional Development Fund (ERDF); Metz Metropole; Airbus GDI Simulation; Fondation CentraleSupélec; Fonds Wetenschappelijk Onderzoek (FWO) (G0E5819N).

\section{References}

1. Marc Sciamanna and Krassimir Panajotov. Route to polarization switching induced by optical injection in verticalcavity surface-emitting lasers. Physical Review A, 73(2):023811, 2006.

2. Martin Virte, Krassimir Panajotov, Hugo Thienpont, and Marc Sciamanna. Deterministic polarization chaos from a laser diode. Nature Photonics, 7(1):60, 2013.

3. Ana Quirce, Cristina de Dios, Angel Valle, and Pablo Acedo. Vcsel-based optical frequency combs expansion induced by polarized optical injection. IEEE Journal of Selected Topics in Quantum Electronics, 25(6):1-9, 2018.

4. Yaya Doumbia, Tushar Malica, Delphine Wolfersberger, Krassimir Panajotov, and Marc Sciamanna. Nonlinear dynamics of a laser diode with an injection of an optical frequency comb. Optics Express, 28(21):30379-30390, 2020.

5. Yaya Doumbia, Tushar Malica, Delphine Wolfersberger, Krassimir Panajotov, and Marc Sciamanna. Optical injection dynamics of frequency combs. Optics Letters, 45(2):435-438, 2020. 